\title{
Apoptotic and genomic effects of corilagin on SKOV3 ovarian cancer cell line
}

This article was published in the following Dove Press journal:

OncoTargets and Therapy

31 March 2017

Number of times this article has been viewed

Rukset Attar'

Zeynep Birsu Cincin ${ }^{2}$

Elif Sinem Bireller ${ }^{3}$

Bedia Cakmakoglu²

'Department of Obstetrics and Gynecology, Yeditepe University Hospital, ${ }^{2}$ Department of Molecular Medicine, Aziz Sancar Institute of Experimental Medicine, Istanbul University, ${ }^{3}$ Department of

Pharmaceutical Microbiology, Faculty of Pharmacy, Istanbul Yeni Yuzyil

University, Istanbul, Turkey
Correspondence: Bedia Cakmakoglu Department of Molecular Medicine, Aziz Sancar Institute of Experimental Medicine, Istanbul University, Capa, Fatih, Istanbul 34093, Turkey

Email bedia@istanbul.edu.tr

\begin{abstract}
Corilagin is a member of the tannin family and has been isolated from traditional Chinese medicinal plants, such as Phyllanthus spp. Corilagin has anti-inflammatory, antioxidative, antiatherogenic, and antihypertensive effects in various experimental models. In this research, we aimed to investigate for the first time whether corilagin had apoptotic and genomic effects in ovarian cancer treatment in the same study. The potential apoptotic of corilagin was investigated using a WST1 cell proliferation test, caspase 3, and mitochondrial membrane potential JC1 assays in a time- and dose-dependent manner. Genomic changes in expression levels against corilagin treatment were measured using an Illumina human HT-12V4 BeadChip microarray. Bioinformatic data analyses were performed using GenomeStudio and Ingenuity Pathway Analysis software. The data of our study demonstrated that there were statistically significant time- and dose-dependent increases in caspase 3 enzymatic activity and loss of mitochondrial membrane potential in line with decreases in cancer cell proliferation. According to gene-ontology analysis, we found that adherens junctions, antigen processing and presentation, and the phosphatidylinositol signaling system were the most statistically significant networks in response to corilagin treatment on SKOV3 cells, in a time- and dose-dependent manner. The apoptotic and genome-wide effects of corilagin on ovarian cancer cells were examined in detail for the first time in the literature. The results of our study suggest that corilagin might have the potential to be used as a new treatment option for epithelial ovarian cancer.
\end{abstract}

Keywords: epithelial ovarian cancer, corilagin, apoptotic effects, gene ontology

\section{Introduction}

Ovarian cancer is one of the leading causes of cancer-related deaths in women around the world. ${ }^{1,2}$ Epithelial ovarian cancer accounts for $\sim 90 \%$ of ovarian cancer cases, with four major subtypes: endometrioid, clear, mucinous, and serous cell. ${ }^{3,4}$ According to recent Globocan (2012) statistical data, 238,700 people were diagnosed as having epithelial ovarian cancer and there were 151,900 deaths worldwide. ${ }^{5}$ Despite various current surgical and therapeutic methods, the 5-year survival ratio of ovarian cancer is nearly $30 \%-40 \%$, because of the absence of significant symptoms at earlier stages and late diagnosis at advanced or metastatic stages. ${ }^{6-10}$ In order to improve the overall survival ratio of patients with ovarian cancer, recent studies have targeted the finding of novel, effective, and nontoxic treatment strategies, as well as nutraceuticals, which are physiologically bioavailable and also cost-effective. ${ }^{11}$

Corilagin belongs to the tannin family, which is extracted from different medicinal plants, including Phyllanthus amarus, $P$. urinaria, Geranium thunbergii, G. carolinianum, and Dimocarpus longan. ${ }^{12-15}$ Recent studies have reported that corilagin has beneficial properties in cardiovascular diseases with its antihypertensive, thrombolytic, and antiatherogenic features. ${ }^{16-18}$ It has also been demonstrated that corilagin can (c)
hereby accept the Terms. Non-commercial uses of the work are permitted without any further permission from Dove Medical Press Limited, provided the work is properly attributed. For permission for commercial use of this work, please see paragraphs 4.2 and 5 of our Terms (https://www.dovepress.com/terms.php). 
serve as a hepatoprotective radical scavenger for superoxide anions and peroxyls. ${ }^{19}$ In addition, recent studies have shown that corilagin has antiproliferative, antitumoral, and antiinflammatory effects. ${ }^{20-23}$ Although the potential therapeutic use of corilagin has already been identified, the underlying molecular mechanism of corilagin remains unclear.

To the best of our knowledge, there are no data about the genomic effects of corilagin in epithelial ovarian carcinoma. In this study, we aimed to investigate antiproliferative and apoptotic effects of corilagin on epithelial ovarian carcinoma using cell culture methods.

\section{Materials and methods}

\section{Cell culture and treatment}

SKOV3 cells line (HTB77) commercially purchased from the American Type Culture Collection were cultured in McCoy's $5 \mathrm{~A}$ modified medium supplemented with fetal bovine serum (heat-inactivated, 10\%) and penicillin-streptomycin (1\%) at $37^{\circ} \mathrm{C}$ in $5 \% \mathrm{CO}_{2} . \mathrm{SKOV} 3$ cells were treated with vehicle $1 \%$ dimethyl sulfoxide (DMSO) alone or 5, 10, 25, 50, and $100 \mu \mathrm{M}$ of corilagin dissolved in 1\% DMSO.

\section{Chemicals and reagents}

McCoy's 5A medium, heat-inactivated fetal bovine serum, and penicillin-streptomycin were purchased from Thermo Fisher Scientific (Waltham, MA, USA). Corilagin was obtained from Sigma-Aldrich (St Louis, MO, USA). The WST1 cell proliferation assay was obtained from Hoffman-La Roche (Basel, Switzerland). The caspase 3 assay was purchased from BioVision (Milpitas, CA, USA). The JC1 mitochondrial membrane potential (MMP) detection assay was obtained from Cayman Chemical (Ann Arbor, MI, USA).

\section{Cell viability assay}

The cell viability effect of corilagin was determined using the WST1 assay. SKOV3 cells were seeded at a density of $10^{4}$ cells/well in 96-well plates. After 24 hours' incubation, cells were treated with $5,10,25,50$, and $100 \mu \mathrm{M}$ corilagin or vehicle $(1 \%, 10 \mu \mathrm{L}$ DMSO) alone as a control for different time periods (24-72 hours). WST1 colorimetric cell proliferation assays were performed after each incubation period. WST1 solution $(10 \mu \mathrm{L})$ was added to each well and incubated for 4 hours at $37^{\circ} \mathrm{C}$. Color development was measured at $450 \mathrm{~nm}$ using a microplate reader (Thermo Fisher Scientific). Cell viability was determined as a percentage of proliferation versus untreated cells (control, 100\%).

\section{Caspase 3 enzymatic activity assay}

Determination of changes in caspase 3 enzymatic activity is known to be one of the important indicators of apoptosis.
We used a caspase 3 colorimetric assay to examine apoptotic changes against corilagin treatment. SKOV3 cells were seeded at a density of $5 \times 10^{5}$ cells/well in 96-well plates. After a 24-hour incubation period, cells were treated with 50 and $100 \mu \mathrm{M}$ corilagin or vehicle (1\% DMSO) alone as a control for different time periods (48-72 hours). After each incubation period, the cells were lysed by adding $50 \mu \mathrm{L}$ chilled cell lysis buffer and incubated on ice for 10 minutes before centrifugation. Supernatant samples were then mixed by adding $50 \mu \mathrm{L}$ reaction buffer, $50 \mu \mathrm{L}$ sample, and $5 \mu \mathrm{L}$ DEVD-pNA substrate, and incubated for 2 hours. The reactions were read at $405 \mathrm{~nm}$ as described in the previous section.

\section{MMP assay}

We used a JC1 assay kit to investigate the loss of MMP in response to corilagin treatment $(50$ and $100 \mu \mathrm{M})$ or vehicle (1\% DMSO) for 48-72 hours in SKOV3 cells (Cayman Chemical). In brief, cells $\left(5 \times 10^{5}\right.$ cells $\left./ 2 \mathrm{~mL}\right)$ were collected by centrifugation at $1,000 \mathrm{rpm}$ for 10 minutes. After removal of supernatant samples, pellets were homogenized in $200 \mu \mathrm{L}$ medium, and $20 \mu \mathrm{L} \mathrm{JC1}$ dye was added to the cells. The cells were then incubated at $37^{\circ} \mathrm{C}$ for 30 minutes, centrifuged, and $200 \mu \mathrm{L}$ assay buffer added to the resulting pellets. All pellets were resuspended in $320 \mu \mathrm{L}$ assay buffer, and $100 \mu \mathrm{L}$ of each was added to a 96-well plate in triplicate. The plate was read using a fluorescence enzyme-linked immunosorbent assay plate reader. Green/ red (485 nm/560 nm) values were calculated to determine changes in MMP.

\section{RNA extraction}

Total RNA was isolated from SKOV3 cells in response to $100 \mu \mathrm{M}$ hesperidin treatment for 72 hours using a "high-pure" RNA isolation kit (Hoffman-La Roche) in accordance with the manufacturer's instructions. DNA concentrations were qualified with NanoDrop spectrophotometry (260:280 nm ratios), and an A260:A280 ratio between 1.8 and 2 was considered suitable for use.

\section{Microarray hybridization}

An HT-12 v4 BeadChip microarray platform of $\sim 47,000$ probes was used to investigate whole-genome expression profile changes in response to corilagin treatment (Illumina, San Diego, CA, USA). After conversion of the totally amplified 500 ng total RNA to complementary DNA, fragmentation and biotin-labeling steps were assessed using a TotalPrep RNA Amplification Kit (Thermo Fisher Scientific). After that, the biotin-labeled complementary RNA sample was hybridized to microarray bead chips as per the manufacturer's protocol. 
The bead chips were scanned using an Illumina BeadArray Reader. Images of bead chips were converted into signal intensities using Illumina GenomeStudio software. This software was also used to detect the quality controls.

\section{Statistical analysis}

Background noise coming from signal intensities of microarray bead chips was corrected and imported into text files using Illumina GenomeStudio. Hierarchical clustering analysis was assessed in data sets to evaluate the proximity between the genes. Hierarchical clusters were constructed using the statistically significant $(P<0.05)$ genes. The related genes were identified as differentially expressed when logarithmic ratios in three different and independent hybridization experiments showed $>1.5$-fold difference in expression level and when the $P$-values were $<0.05$. The Kyoto Encyclopedia of Genes and Genomes, Ingenuity Pathway Analysis (IPA) tools, gene ontology, and pathway analyses were used to ascertain biologic meanings for the differentially expressed genes between the data points.

\section{Results}

\section{Corilagin inhibited SKOV3-cell proliferation in a time- and dose- dependent manner}

We used the WST1 colorimetric cell proliferation assay to determine cytotoxic effects of corilagin on SKOV3 ovarian cancer cells. The cells were incubated with increasing concentrations $(5-100 \mu \mathrm{M})$ of corilagin for 24,48 , and 72 hours, and then treated with the WST1 assay after each incubation period. The results showed that 50 and $100 \mu \mathrm{M}$ corilagin treatment had significant effects in a time-dependent manner $(P<0.05, P<0.01)$. Half-maximal inhibitory-concentration values of corilagin for 24,48 , and 72 hours were calculated from both cell proliferations and found to be $0.7 \mathrm{mM}$ (Figure 1).

\section{Corilagin increased caspase 3 enzymatic activity in a time- and dose-dependent manner}

To determine the apoptotic effects of corilagin on SKOV3 cells, cells were incubated with 50 or $100 \mu \mathrm{M}$ of corilagin for 48-72 hours, and then changes in caspase 3 enzymatic activity were determined. There was a 1.47-fold increase in caspase 3 enzymatic activity in response to 48 hours with $50 \mu \mathrm{M}$ corilagin. We also found that there was a 2.58 -fold increase at 48 hours and a 2.08 -fold increase at 72 hours in caspase 3 enzymatic activity in response to treatment with $100 \mu \mathrm{M}$ corilagin (Figure 2).

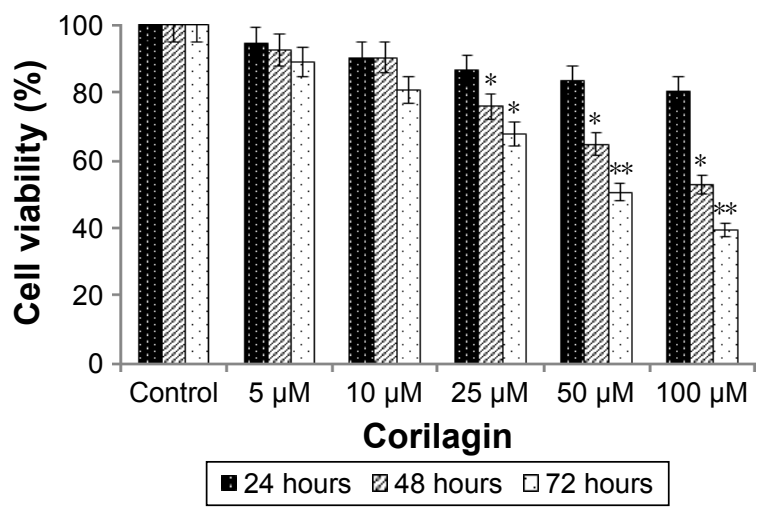

Figure I Effects of corilagin on cell proliferation.

Notes: WSTI proliferation was performed using triplicate samples in three independent experiments. Statistical significance was determined using two-way analysis of variance, and $P<0.05$ was considered significant $(* P<0.05 ; * * P<0.01)$.

\section{Corilagin induced loss of MMP in a time- and dose-dependent manner}

To determine the loss of MMP, cells were incubated with 50 or $100 \mu \mathrm{M}$ of corilagin for $48-72$ hours. The results showed that there was a 1.89-fold increase in the loss of MMP in response to $50 \mu \mathrm{M}$ corilagin compared with untreated cells. We also found that there was a 2.1-fold increase at 48 hours and 1.89-fold increase at 72 hours in caspase 3 enzymatic activity in response to treatment with $100 \mu \mathrm{M}$ corilagin (Figure 3 ).

\section{Corilagin induced changes in gene expression profiles}

To investigate changes in whole-genome expression levels in response to corilagin treatment ( 72 hours, $100 \mu \mathrm{M})$, we used HT-12 v4 BeadChip microarray profiling. After analyzing

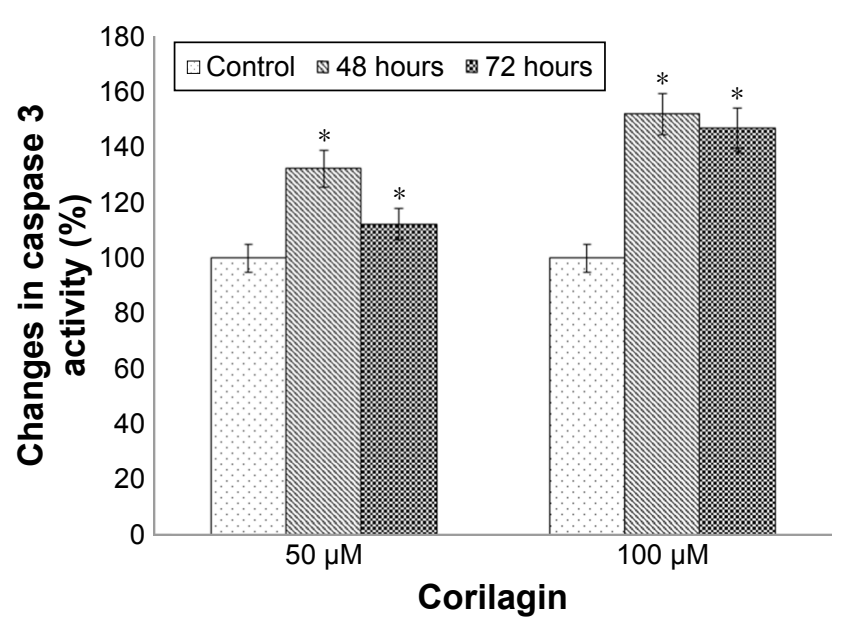

Figure 2 Effects of corilagin on caspase 3 enzymatic activity in SKOV3 cells. Notes: Changes in caspase 3 enzymatic activity in response to increasing concentrations of corilagin in SKOV3 cells. The results are the means of two independent experiments. $* P<0.05$ was considered significant. 


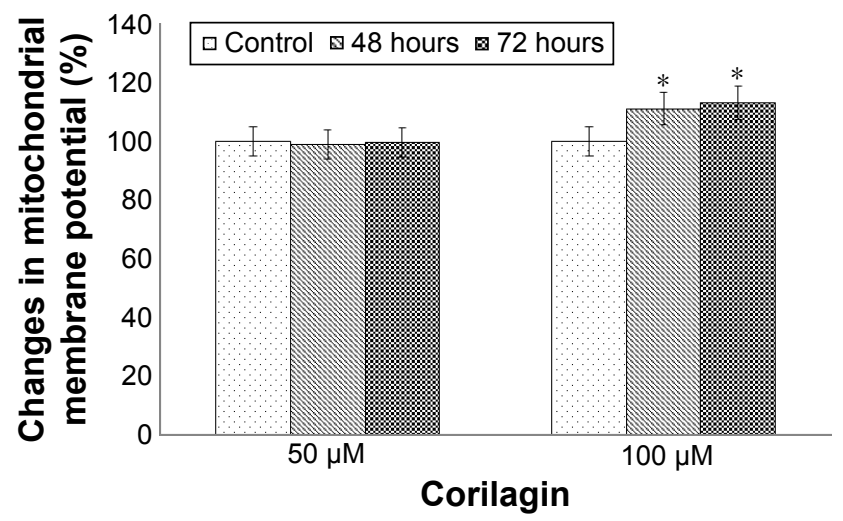

Figure 3 Effects of corilagin on membrane potential in SKOV3 cells.

Notes: Loss of mitochondrial membrane potential in response to increasing concentrations of corilagin in SKOV3 cells. The results are presented as the means of two independent experiments. $* P<0.05$ was considered significant.

the most statistically significant transcripts, we clustered the data based on their expression patterns. In addition, biofunctional annotation analysis was performed to find the downstream pathways in response to corilagin treatment. We also used IPA analysis to understand the relationship between pathways in accordance with the literature (Figure 4 and Table 1). Based on IPA analysis, three major groups of pathways were shown as statistically significant in corilagin-induced SKOV3 cells: adherens junctions, antigen processing and presentation, and the phosphatidylinositol signaling system (Table 2).

\section{Discussion}

The antiproliferative, apoptotic, and genomic effects of corilagin in epithelial ovarian cancer were examined in detail for the first time in the literature. The results of our study suggest that corilagin has an antitumor effect on ovarian cancer cells and has potential for use as a new treatment for epithelial ovarian cancer.

Hau et al investigated the antitumoral effects of corilagin on HEP3B hepatocellular carcinoma cell lines in an athymic xenograft mouse model. They found significant inhibition in tumor growth in response to corilagin treatment. ${ }^{20}$ Furthermore, they also indicated that corilagin had no adverse effects on the liver, based on alanine transaminase and aspartate aminotransferase tests. Gambari et al also investigated the sensitization of HEP3B cells to corilagin-induced cisplatin and doxorubicin effects, and showed that using low-dose corilagin could be used in combination with cisplatin and doxorubicin to increase antitumoral effects of chemotherapy treatment. ${ }^{21}$ In addition to these studies, Ming et al also examined the effects of corilagin on the relationship between antitumoral mechanism and cell cycle proteins using BEL7402 and SMMC7721 hepatocellular carcinoma cells. ${ }^{22}$ They showed that corilagin-induced SMMC7721 cells arrested at the $\mathrm{G}_{2} / \mathrm{M}$ phase in response to corilagin via downregulation of the pAkt pathway and upregulation of p53. Together, these data indicate that corilagin has potential for use as a novel antitumoral drug for hepatocellular cancer treatment. ${ }^{22}$

In addition to its antitumoral effects, two reports emphasized the reparative effects of corilagin for lung injury caused by tobacco products and aerosols. Muresan et al demonstrated that cigarette smoking stimulated the loss of intercellular junctions of CALU3 lung epithelial cells, and

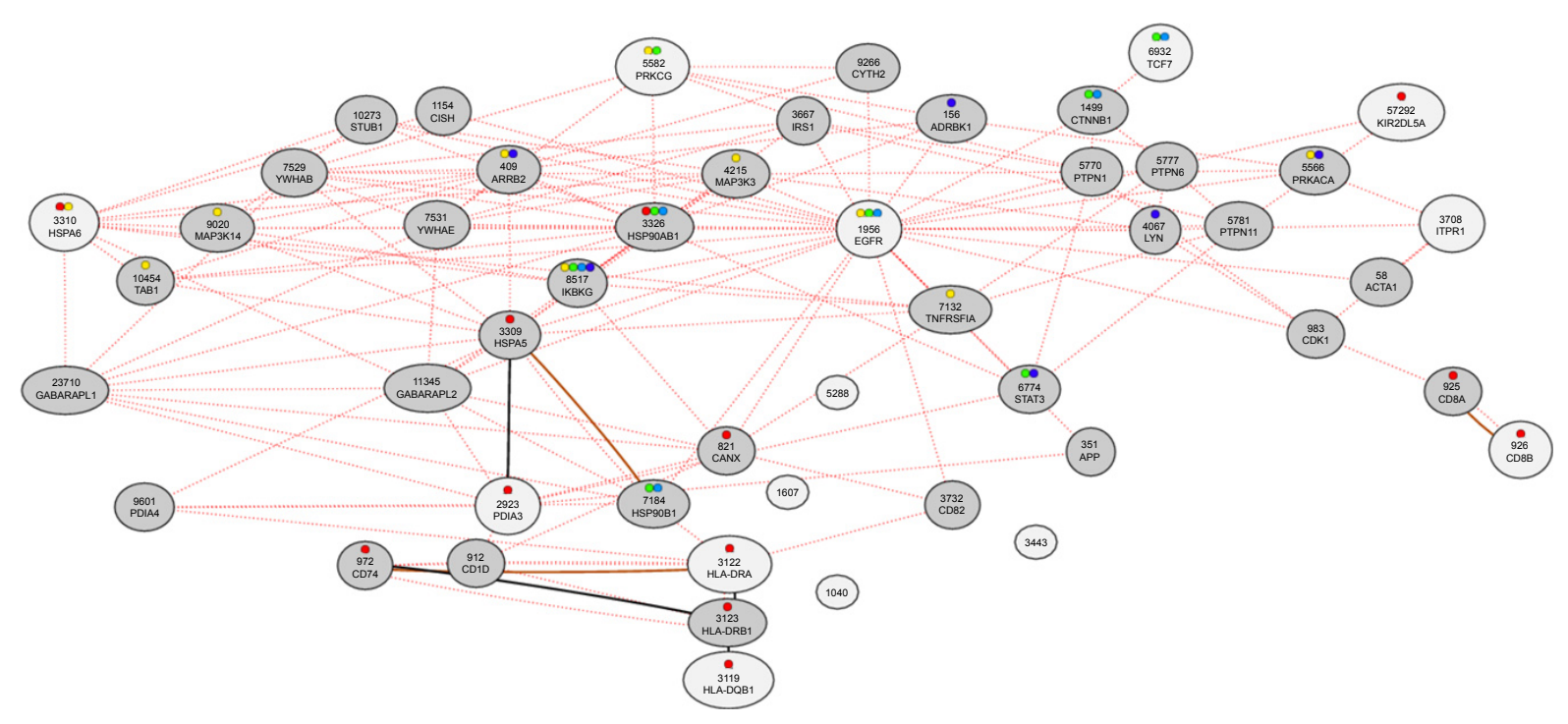

Figure 4 Top networks and gene connections.

Notes: Literature-based clustering of differentially expressed genes using Ingenuity Pathway Analysis (IPA) after 78 hours of I00 $\mu$ M corilagin stimulation in SKOV 3 cells. According to IPA, three major pathways emerged: antigen processing and presentation, a MAPK signaling pathway, and a chemokine signaling pathway. 
Table I Top networks and gene connections

\begin{tabular}{llll}
\hline KEGG ID & Title & Genes, $\mathbf{n}$ & $\begin{array}{c}\text { Link to the KEGG } \\
\text { map (multiple genes) }\end{array}$ \\
\hline hsa046I2 & Antigen processing and presentation & 12 & 0 \\
hsa04010 & MAPK signaling pathway & 10 & 0 \\
hsa05200 & Pathways in cancer & 8 & 6 \\
hsa05206 & Ovarian cancer & 6 & 0 \\
hsa04062 & Chemokine signaling pathway & 6 & \\
\hline
\end{tabular}

Notes: Literature-based clustering of differentially expressed genes using Ingenuity Pathway Analysis (IPA) after 78 hours of I00 $\mu$ M corilagin stimulation in SKOV3 cells. According to IPA, three major pathways emerged: antigen processing and presentation, a MAPK signaling pathway, and a chemokine signaling pathway.

Abbreviation: KEGG, Kyoto Encyclopedia of Genes and Genomes.

corilagin reversed these changes by stimulating expression of connexins. ${ }^{23}$ Wang et al investigated the effects of corilagin in a pulmonary fibrosis model, and found that high-dose corilagin could downregulate expressions of malondialdehyde, IKK $\alpha$, pIKK $\alpha$, NFкB p65, TNF $\alpha$, and IL-1 $\beta$ to abolish the adverse effects of bleomycin exposure. ${ }^{24}$ They also showed that TGF $\beta$ production and $\alpha$-SMA expression were inhibited by corilagin treatment. In light of these data, it could be speculated that corilagin could repair lung epithelial injury by affecting cytokine release and the TGF $\beta_{1}$ signaling pathway. ${ }^{24}$

To the best of our knowledge, no reports have investigated the apoptotic and genomic effects of corilagin on ovarian cancer cells in the same study. However, one report showed the anticarcinogenic effect of corilagin on SKOV3IP, HEY, and HO8910PM ovarian cancer cell lines and a xenograft model using flow cytometry and protein arrays..$^{25}$ The authors found that corilagin-induced ovarian cancer cells arrested at the $\mathrm{G}_{2} / \mathrm{M}$ cell cycle point, which correlates with the data from Ming et al. Furthermore, they also showed that levels of cyclin B1, Myt1, and phospho-CDC2 were downregulated in response to corilagin treatment using immunoblotting. In addition, supernatant samples of corilagin-induced ovarian

Table 2 Genes involved in top network connections

\begin{tabular}{lll}
\hline Pathway name & Differentially expressed genes & $\begin{array}{l}\text { Adjusted } \\
\boldsymbol{P} \text {-value }\end{array}$ \\
\hline Adherens junction & EGFR, TCF7 & $9^{-15}$ \\
Antigen processing & CD8B, HLA-DQBI, HLA-DRA, & $3.7^{-14}$ \\
and presentation & HSPA6, IFNA6, KIR2DL5A, PDIA3 & \\
$\begin{array}{l}\text { Phosphatidylinositol } \\
\text { signaling system }\end{array}$ & CDSI, DGKB, ITPRI, PIK3C2G, & $4.7^{-6}$ \\
\hline
\end{tabular}

Notes: Literature-based clustering of differentially expressed genes using Ingenuity Pathway Analysis (IPA) after 78 hours of $100 \mu \mathrm{M}$ corilagin stimulation in SKOV3 cells. According to KEGG, three major pathways emerged: adherens junctions, antigen processing and presentation, and a phosphatidylinositol signaling system were the most statistically significant networks in response to corilagin treatment. Abbreviation: KEGG, Kyoto Encyclopedia of Genes and Genomes. cancer cells have shown inhibition for TGF $\beta$ secretion and blockage for TGF $\beta$-induced Snail stabilization. According to that study, corilagin specifically targeted TGF $\beta$ secretion to block both canonical SMAD and noncanonical ERK-Akt pathway activation.

In this study, we investigated the apoptotic and genomic effects of corilagin on SKOV3 epithelial ovarian carcinoma cells. We performed WST1, caspase 3, and JC1 assays to understand the dose- and time-dependent effects of corilagin on SKOV3 cancer-cell proliferation and viability. Our results showed correlation with studies that used hepatocellular and ovarian cancer cell lines. We also reported that corilagininduced inhibition of SKOV3 cell proliferation showed correlation with increased apoptotic cancer cells. In addition, we found that corilagin stimulated the MAPK and phosphatidylinositol signaling system to show its antitumoral effects on downstream pathways. These data show that corilagin has potential anticarcinogenic and antitumoral effects, and thus has potential for use as a novel drug for ovarian cancer treatment. Further studies will help to understand the mechanism of the corilagin-induced effect on cellular metabolism.

\section{Acknowledgments}

This work was funded by Istanbul University Scientific Research Project 33202. We thank Mr David F Chapman for editing the manuscript.

\section{Disclosure}

The authors report no conflicts of interest in this work.

\section{References}

1. Jemal A, Siegel R, Xu J, Ward E. Cancer statistics, 2010. CA Cancer J Clin. 2010;60(5):277-300.

2. Ren F, Shen J, Shi H, Hornicek FJ, Kan Q, Duan Z. Novel mechanisms and approaches to overcome multidrug resistance in the treatment of ovarian cancer. Biochim Biophys Acta. 2016;1866(2):266-275.

3. Cannistra SA. Cancer of the ovary. N Engl J Med. 2004;351(24): 2519-2529. 
4. Deng J, Wang L, Chen H, et al. Targeting epithelial-mesenchymal transition and cancer stem cells for chemoresistant ovarian cancer. Oncotarget. 2016;7(34):55771-55788.

5. Torre LA, Bray F, Siegel RL, Ferlay J, Lortet-Tieulent J, Jemal A. Global cancer statistics, 2012. CA Cancer J Clin. 2015;65(2):87-108.

6. Cakmakoglu BA, Attar R, Kahraman OT, et al. Cyclooxygenase-2 gene and epithelial ovarian carcinoma risk. Mol Biol Rep. 2011;38(5): 3481-3486.

7. Baldwin LA, Huang B, Miller RW, et al. Ten-year relative survival for epithelial ovarian cancer. Obstet Gynecol. 2012;120(3):612-618.

8. Qin D, Wang W, Lei H, et al. CDDO-Me reveals USP7 as a novel target in ovarian cancer cells. Oncotarget. 2016;7(47):77096-77109.

9. Vaughan S, Coward JI, Bast RC Jr, et al. Rethinking ovarian cancer: recommendations for improving outcomes. Nat Rev Cancer. 2011; 11(10):719-725.

10. Qin DJ, Tang CX, Yang L, et al. Hsp90 is a novel target molecule of CDDO-Me in inhibiting proliferation of ovarian cancer cells. PLoS One. 2015;10(7): 0132337.

11. Banerjee S, Kaye SB. New strategies in the treatment of ovarian cancer: current clinical perspectives and future potential. Clin Cancer Res. 2013;19(5):961-968.

12. Hau DK, Gambari R, Wong RS, et al. Phyllanthus urinaria extract attenuates acetaminophen induced hepatotoxicity: involvement of cytochrome P450 CYP2E1. Phytomedicine. 2009;16(8):751-760.

13. Shen ZQ, Dong ZJ, Peng H, Liu JK. Modulation of PAI-1 and tPA activity and thrombolytic effects of corilagin. Planta Med. 2003;69(12): 1109-1112.

14. Sudjaroen Y, Hull WE, Erben G, et al. Isolation and characterization of ellagitannins as the major polyphenolic components of longan (Dimocarpus longan Lour) seeds. Phytochemistry. 2012;77:226-237.

15. Okabe S, Suganuma M, Imayoshi Y, Taniguchi S, Yoshida T, Fujiki H. New TNF- $\alpha$ releasing inhibitors, geraniin and corilagin, in leaves of Acer nikoense, megusurinoki. Biol Pharm Bull. 2001;24(10):1145-1148.
16. Duan W, Yu Y, Zhang L. Antiatherogenic effects of Phyllanthus emblica associated with corilagin and its analogue. Yakugaku Zasshi. 2005; 125(7):587-591.

17. Cheng JT, Lin TC, Hsu FL. Antihypertensive effect of corilagin in the rat. Can J Physiol Pharmacol. 1995;73(10):1425-1429.

18. Duan WG, Shen ZQ, Yan M, Yun Y, Zhang LY. Corilagin, a promising natural product to treat cardiovascular diseases. In: Singh VK, Mishra SK, Govil JN, editors. Phytopharmacology and Therapeutic Values II. Houston: Studium Press; 2008:163-172.

19. Kinoshita S, Inoue Y, Nakama S, Ichiba T, Aniya Y. Antioxidant and hepatoprotective actions of medicinal herb, Terminalia catappa $\mathrm{L}$. from Okinawa Island and its tannin corilagin. Phytomedicine. 2007;14(11): 755-762.

20. Hau DK, Zhu GY, Leung AK, et al. In vivo anti-tumour activity of corilagin on HEP3B hepatocellular carcinoma. Phytomedicine. 2010; 18(1):11-15.

21. Gambari R, Hau DK, Wong WY, Chui CH. Sensitization of HEP3B hepatoma cells to cisplatin and doxorubicin by corilagin. Phytother Res. 2014;28(5):781-783.

22. Ming Y, Zheng Z, Chen L, et al. Corilagin inhibits hepatocellular carcinoma cell proliferation by inducing G2/M phase arrest. Cell Biol Int. 2013;37(10):1046-1054.

23. Muresan XM, Cervellati F, Sticozzi C, et al. The loss of cellular junctions in epithelial lung cells induced by cigarette smoke is attenuated by corilagin. Oxid Med Cell Longev. 2015;2015:631758.

24. Wang Z, Guo QY, Zhang XJ, et al. Corilagin attenuates aerosol bleomycin-induced experimental lung injury. Int J Mol Sci. 2014;15(6): 9762-9779.

25. Jia L, Jin H, Zhou J, et al. A potential anti-tumor herbal medicine, corilagin, inhibits ovarian cancer cell growth through blocking the TGF- $\beta$ signaling pathways. BMC Complement Altern Med. 2013;13:33.
OncoTargets and Therapy

\section{Publish your work in this journal}

OncoTargets and Therapy is an international, peer-reviewed, open access journal focusing on the pathological basis of all cancers, potential targets for therapy and treatment protocols employed to improve the management of cancer patients. The journal also focuses on the impact of management programs and new therapeutic agents and protocols on

\section{Dovepress}

patient perspectives such as quality of life, adherence and satisfaction The manuscript management system is completely online and includes a very quick and fair peer-review system, which is all easy to use. Visit http://www.dovepress.com/testimonials.php to read real quotes from published authors. 